\title{
Blocking the Receptor for Advanced Glycation End Product Activation Attenuates Autoimmune Myocarditis
}

\author{
Woo-In Yang, MD; Dajeong Lee; Da Lyung Lee, PhD; Sung-Yu Hong, PhD; \\ Sang-Hak Lee, MD, PhD; Seok-Min Kang, MD, PhD; Dong-Hoon Choi, MD, PhD; \\ Yangsoo Jang, MD, PhD; Se Hoon Kim, MD, PhD; Sungha Park, MD, PhD
}

\begin{abstract}
Background: The Receptor for Advanced Glycation End Products (RAGE) is a pattern recognition receptor for endogenous ligands, and is associated with various inflammatory diseases. However, the role of RAGE activation in myocarditis has yet to be examined. The potential role of RAGE in the development of experimental autoimmune myocarditis (EAM) and the effect of RAGE blocking in attenuating the inflammation in the EAM was investigated.

Methods and Results: EAM was evoked in Lewis rats by immunization with porcine cardiac myosin. Soluble RAGE (sRAGE) was injected to block RAGE activation. Echocardiogram, histological, and immunohistochemical examinations were conducted on days 21 and 42. In rats with EAM, RAGE expression in cardiac tissue was prominent on day 21. Rats administered SRAGE during the early antigen-priming phase showed marked attenuation in acute and chronic inflammation compared with untreated rats. RAGE expression was significantly reduced in rats treated in the early phase. However, sRAGE administration, after the initial antigen-priming phase, failed to ameliorate EAM development.
\end{abstract}

Conclusions: RAGE expression was significantly increased in the heart during EAM. Blocking RAGE activation with sRAGE during the early antigen-priming phase reduced acute and chronic inflammation and improved cardiac function. In contrast, blocking RAGE after the early phase did not attenuate EAM development. These results imply that RAGE is involved in regulating innate immune responses during the early phase of myocarditis development. (Circ J 2014; 78: 1197-1205)

Key Words: Immunology; Myocarditis; The Receptor for Advanced Glycation End Products

$\mathbf{M}$ yocarditis is an often fatal, inflammatory disease of the heart with no known specific treatment. Clinical manifestations range from asymptomatic presentation to development of heart failure, arrhythmia, or circulatory collapse. ${ }^{1-4}$ The chronic phase of myocarditis might result in the development of dilated cardiomyopathy in up to $20 \%$ of patients. ${ }^{1-4}$ Although viral infection is a frequent cause of myocarditis, the autoimmune response has been regarded to be important in disease progression..$^{5,6}$ The exposure of cardiac autoantigen to the host immune system with or without viral persistence has been suggested to be crucial for myocardial inflammation. Experimental autoimmune myocarditis (EAM) is a widely used experimental model of myocarditis, which is induced by autoimmunization. After cardiac myosin injection, rats present with severe myocardial damage with inflammatory cell infiltration similar to human myocarditis. ${ }^{7,8}$ In EAM models, cardiac autoantigens strongly induce innate immune responses through activation of Toll-like receptors (TLRs) and inflammasomes, resulting in marked upregulation of pro-inflammatory cytokines. ${ }^{9}$ Subsequently, cell-mediated immunity

Received October 10, 2013; revised manuscript received December 9, 2013; accepted January 14, 2014; released online March 6, 2014 Time for primary review: 35 days

Department of Cardiology, CHA Bundang Medical Center, CHA University, Sungnam (W.-I.Y.); Cardiovascular Research Institute (D.L.), Cardiovascular Product Evaluation Center, Cardiovascular Research Institute (D.L.L., S.-Y.H.), Cardiology Division, Severance Cardiovascular Hospital (S.-H.L., S.-M.K., D.-H.C., Y.J., S.P.), and Department of Pathology (S.H.K.), Yonsei University College of Medicine, Seoul, Republic of Korea

Drs. Se Hoon Kim and Sungha Park contributed equally as corresponding authors to this study.

Mailing address: Sungha Park, MD, PhD, Cardiology Division, Yonsei University College of Medicine, 50 Yonsei-ro, Seodaemun-gu, Seoul 120-752, Korea. E-mail: shpark0530@yuhs.ac

Mailing address: Se Hoon Kim, MD, PhD, Department of Pathology, Yonsei University College of Medicine, 50 Yonsei-ro, Seodaemun-gu, Seoul 120-752, Korea. E-mail: paxco@yuhs.ac

ISSN-1346-9843 doi:10.1253/circj.CJ-13-1235

All rights are reserved to the Japanese Circulation Society. For permissions, please e-mail: cj@j-circ.or.jp 
by cytotoxic T-cells and macrophages plays an important role during the peak inflammatory phase.

The Receptor for Advanced Glycation End Products (RAGE) is a multi-ligand member of the immunoglobulin superfamily of cell surface molecules. ${ }^{10,11}$ RAGE activation by RAGE ligands is associated with the induction of oxidative stress, increases in inflammatory cytokines, and recruitment of pro-inflammatory cells. ${ }^{12}$ RAGE and ligand interactions modulate gene expression and cellular properties via various signal pathway such as MAPK, SAPK/JNK, Rho GTPase and JAK/SATA. ${ }^{13,14}$ Consequently, ligation of RAGE activates NF-kB causing immune responses and tissue damage. ${ }^{14,15}$ In myocarditis, self-antigens have been known to activate an immune response through TLRs signaling. ${ }^{16}$ As RAGE is an important endogenous pattern-recognition receptor that triggers innate immune responses, self-proteins generated during myocarditis might activate RAGE and result in inflammation. However, there have been no published reports examining the role of RAGE in myocarditis. Therefore, in the present study, we sought to investigate the extent to which RAGE-mediated inflammation contributes to the development of EAM. Furthermore, as previous studies had shown that soluble RAGE (sRAGE) could suppress inflammation by the blockade of RAGE receptor signaling, ${ }^{17-19}$ we evaluated the potential therapeutic efficacy of sRAGE administration during the development of EAM.

\section{Methods}

\section{Animals and Immunization}

Male Lewis rats (6 weeks old, 200-250g) were purchased from Sankyo Laboratory (Tokyo, Japan). After 1 week, they (7-weeks-old, 250-300 g) were immunized with purified porcine cardiac myosin to induce EAM. ${ }^{7}$ The rats were maintained in compliance with animal welfare guidelines of the Institute of Experimental Animals, Yonsei University College of Medicine. Purified porcine cardiac myosin (Sigma Chemical $\mathrm{Co}$, St. Louis, MO, USA) was dissolved in $0.01 \mathrm{~mol} / \mathrm{L}$ phosphate-buffered saline and emulsified with an equal volume of complete Freund's adjuvant (CFA) (Difco, Sparks, MD, USA), supplemented with Mycobacterium tuberculosis H37RA (Difco) at a concentration of $10 \mathrm{mg} / \mathrm{ml}$. On days 0 and 7 , rats were injected subcutaneously in the footpads with $0.2 \mathrm{ml}$ of emulsion, yielding an immunizing dose of $1.0 \mathrm{mg}$ of cardiac myosin per rat. Rats in the normal control group were injected only with $0.2 \mathrm{ml}$ of CFA. This study protocol was reviewed and approved by the Institutional Animal Care and Use Committee of Yonsei University College of Medicine.

\section{Reagents}

We used sRAGE conjugated to the Fc potion of immunoglobulin for our experiments (A\&R Therapeutics, Seoul, Korea). Conjugated sRAGE was administered daily at a dose of $5 \mu \mathrm{g}$ by intraperitoneal injection according to the specified regimen in each study group.

\section{Experiment Protocol}

Experiment 1 This experiment was carried out to investigate the inflammatory phase of EAM and confirm the potential role of RAGE in the pathogenesis of EAM. Rats with EAM (immunized with cardiac myosin, $n=51$ ) were prepared. An echocardiographic examination was performed on days 0,11 , $14,21,28,35$, and 42 after myosin injection. Rats $(n=5$ on day $0, n=7$ on day $11, n=7$ on day $14, n=8$ on day $21, n=8$ on day $28, n=8$ on day $35, n=8$ on day 42 ) were sacrificed after an echocardiographic examination for the pathologic study. As previous studies demonstrated that myocardial inflammation began at approximately 11-14 days, we performed evaluations on day 11 , not on day 7 .

Experiment 2 This experiment was carried out to investigate the effect of blocking RAGE signaling on EAM. To identify potential effects against progressing or established disease, sRAGE injection was administered at different time intervals according to the various immunologic phases of EAM. As it had been demonstrated that initiation of myocardial inflammation begins at approximately 11-14 days, we defined days 0-11 as the antigen-priming phase, days $12-20$ as the immune response phase, and day 20 and beyond as the chronic recovery phase. ${ }^{8,20}$ Rats with EAM were divided into 5 groups according to the sRAGE injection time: (a) Group R 0-20 (from day 0 to day 20, n=11); (b) Group R 0-11 (from day 0 to day 11, $n=12$ ); (c) Group R 12-20 (from day 12 to day 20, n=11); (d) Group R 21-35 (from day 21 to day 35, $n=6$ ); and (e) Group $C$ (untreated group, $n=12$ ). Rats without EAM (Group N, n=6) were also included. sRAGE was administered by intraperitoneal injection. An echocardiographic examination was conducted on days 21 (Group N, n=3; Group R 0-20, n=6; Group R 0-11, $\mathrm{n}=6$; Group R 12-20, n=6; Group C, n=4) and days 42 (Group $\mathrm{N}, \mathrm{n}=3$; Group R 0-20, n=5; Group R 0-11, n=6; Group R 12$20, n=5$; Group R 21-35, n=6; Group C, n=8). After the echocardiographic examination, rats were sacrificed for the pathologic study.

\section{Echocardiographic Examination}

Transthoracic echocardiography was performed on rats anesthetized by intraperitoneal administration of pentobarbital sodium (0.25 mg/kg; Dainihon Chemical Co, Osaka, Japan). An echocardiographic machine with a $15 \mathrm{MHz}$ transducer (Vivid Q; General Electric-Vingmed, Milwaukee, WI, USA) was used. A M-mode echocardiogram was obtained along the shortaxis view of the left ventricle at the papillary muscles, and the left ventricular ejection fraction (LVEF) was calculated from M-mode echocardiograms. ${ }^{21}$

\section{Heart Preparation}

After the echocardiographic examination on days 21 and 42, the heart was extracted above the origin of great vessels. Then, the heart was divided into 5 transverse sections. Three sections from the apex, mid-ventricular, and basal levels were prepared for pathologic examination.

\section{Histologic Evaluation}

Myocardial tissues were fixed in $10 \%$ neutral formaline, embedded in paraffin and sectioned. The sections were stained by hematoxylin-eosin and Masson's trichrome. The extent of fibrosis was measured with a digital camera (Olympus DP72; Tokyo, Japan) and a computer-assisted analyzer (Image J 1.43 Software Package downloaded from http//imagej.nih.gov/ij/; NIH, Bethesda, MD, USA). The blue-stained area and total myocardial area were analyzed with color-based thresholding. The area of fibrosis (percentage value of the blue-stained area/ entire area) was calculated by 2 investigators who were blinded to slide identification. The average of the area ratio in the 3 sections was calculated.

\section{Immunohistochemical Study}

Immunohistochemistry was used to examine CD45RC, CD4RC, CD8RC, RAGE, high-mobility group box 1 protein (HMGB1) and intracellular adhesion molecule-1 (ICAM-1) expression in the myocardium. We performed immunohistochemical staining with anti-CD45RC (1:500, ab33945; Abcam, Cambridge, 
UK), CD4RC (1:500, ab82252; Abcam, Cambridge, UK), CD8RC (1:500, ab65865; Abcam, Cambridge, UK), RAGE (1:50, ab3611; Abcam, Cambridge, UK), HMGB1 (1:100, ab18256; Abcam Cambridge, UK) and ICAM-1 (1:50, ab33894; Abcam, Cambridge, UK) antibodies using a Ventana BenchMark XT autostainer (Ventana Medical System, Inc, Tuscon, AZ, USA), according to the manufacturer's protocol. The areas of CD45, RAGE, HMGB1 and ICAM-1 immunoreactivity were analyzed with a digital camera (Olympus DP72, Tokyo, Japan) and image-processing software (Image J 1.43; Bethesda, MD, USA). The stained area and total myocardial area were analyzed with color-based thresholding. The affected area (percentage value of stained area/entire area) was calculated by 2 investigators who were blinded to the slide identification. The average of the area ratio in the 3 sections was calculated.

\section{Double Immunofluorescence Staining}

We conducted double immunofluorescence staining for RAGE with CD68, and HMGB1 with CD68, on myocarditis tissues. After deparaffinization, the slides were blocked by using $3 \%$ $\mathrm{H}_{2} \mathrm{O}_{2}$ and $3 \mathrm{mg} / \mathrm{ml}$ donkey serum for $1 \mathrm{~h}$ at room temperature. After the incubation of mouse CD68 (1:100, abcam \#ab31630) for $90 \mathrm{~min}$ at room temperature, we incubated mouse $\mathrm{Cy} 3$ (Jackson immunoResearch \#715-165-151) for $60 \mathrm{~min}$ at room temperature. After washing, we incubated rabbit RAGE (1:100, abcam \#ab3611) and rabbit HMGB1 (1:100, abcam \#ab18256) for $90 \mathrm{~min}$ at room temperature). Subsequently, incubation with Rabbit Alexa 488 (Jackson immunoResearch \#715-545152) was conducted for $60 \mathrm{~min}$ at room temperature and the slides were observed under a fluorescence microscope.

\section{Enzyme-Linked Immunosorbent Assay of RAGE and HMGB1} An ELISA was performed to determine serum levels of interleukin-1 $\beta$ (IL-1 $\beta$ ), RAGE, and HMGB1. Blood was obtained from the abdominal aorta after animals were sacrificed for pathologic examination. Serum IL-1 $\beta$, RAGE and HMGB1

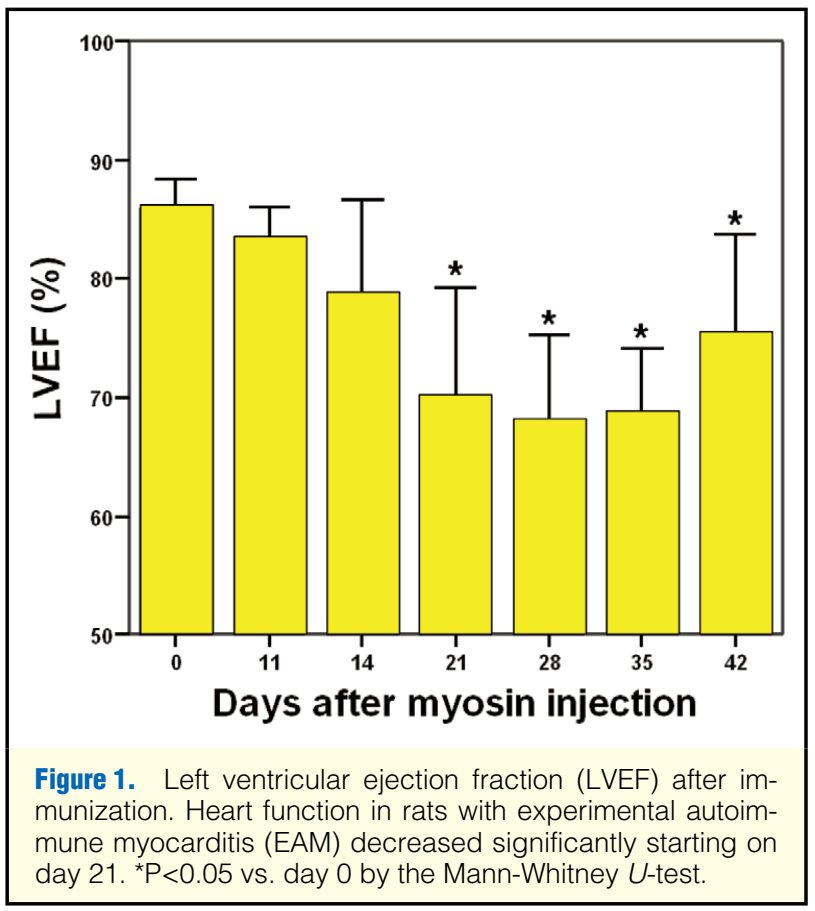

levels were quantified using the commercial kits (R\&D systems, Minneapolis, MN, USA; Abcam, Cambridge, UK; IBL International, Hamburg, Germany) according to the manufacturer's instructions.

\section{Statistical Analysis}

All data were expressed as the mean \pm standard deviation (SD). Comparisons between sRAGE-treated groups and untreated groups were performed by using the Mann-Whitney $U$-test. Data analyses were conducted with a commercially available
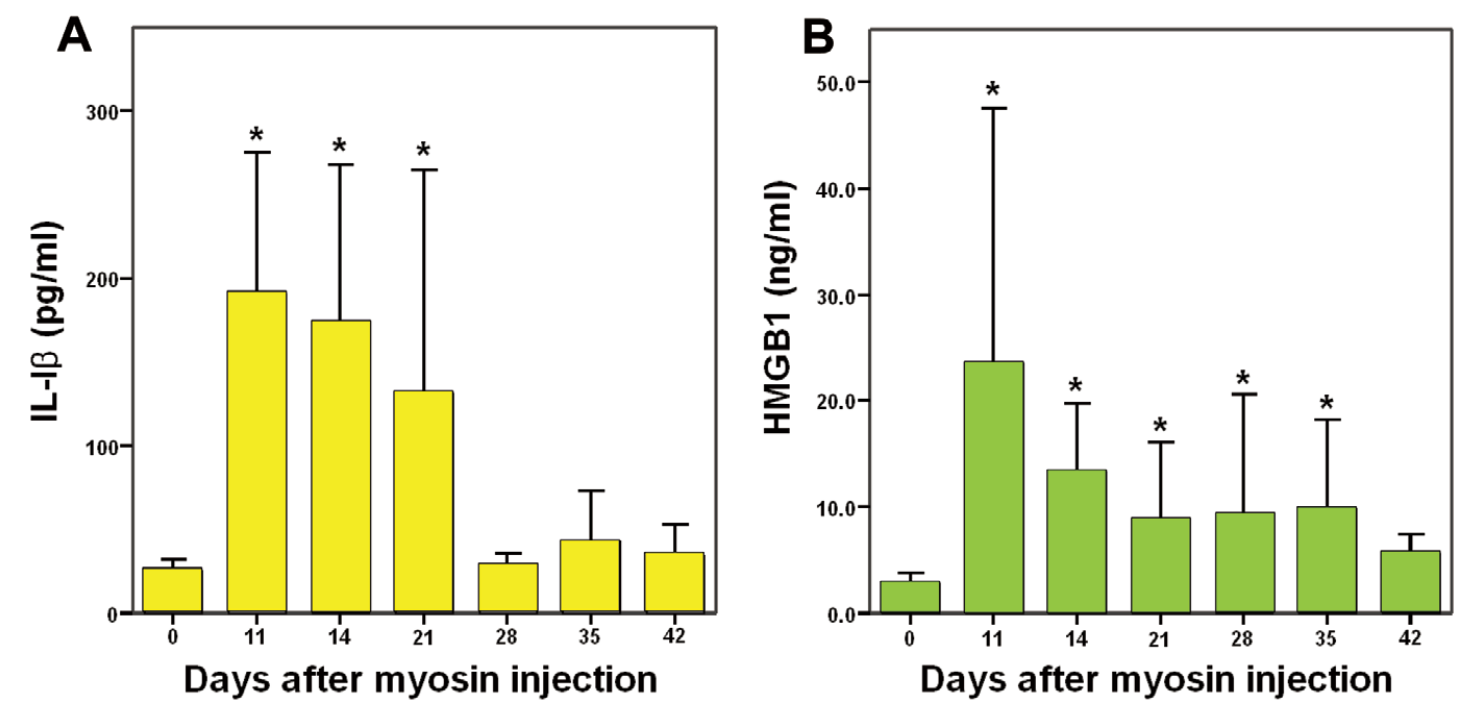

Figure 2. Serum levels of interleukin (IL)-1 $\beta$ and high-mobility group box 1 protein (HMGB1) in rats with experimental autoimmune myocarditis (EAM) after immunization. (A) Serum levels of IL-1 $\beta$ were markedly elevated from day 11 to day 21 in the EAM model system. (B) Serum levels of HMGB1 peaked on day 11. ${ }^{*} P<0.05$ vs. day 0 by the Mann-Whitney $U$-test. 


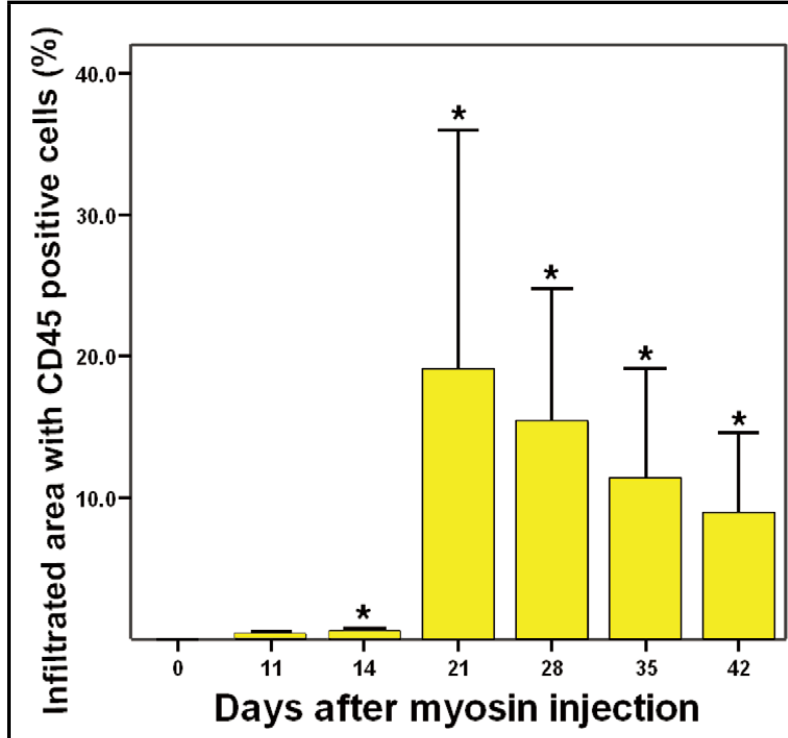

Figure 3. Active inflammation measured by the infiltration of CD45-positive cells after induction of experimental autoimmune myocarditis (EAM) in rats; myocardial infiltration of CD45-positive cells began on day 14 and became prominent on day 21 . ${ }^{*} \mathrm{P}<0.05$ vs. day 0 by the Mann-Whitney $U$ test.

statistics software package (SPSS 18, Chicago, IL, USA). A P-value of $<0.05$ was considered statistically significant.

\section{Results}

\section{Reduced Cardiac Function in EAM}

The heart function in rats with EAM was reduced compared to that of controls beginning at day 14 , with a marked reduction of the LVEF from day 21 to day 42 (Figure 1).

\section{Elevated Serum Level of IL-1 $\beta$ and HMGB1 in EAM}

After the induction of EAM, serum levels of the pro-inflammatory cytokine, IL-1 $\beta$, were significantly elevated from day 11 to day 21 . After this point, serum IL-1 $\beta$ concentrations re- turned to baseline levels (Figure 2A). Serum HMGB1 concentrations increased markedly by day 11 and remained elevated through to day 35 (Figure 2B).

\section{Prominent Inflammation in Myocarditis}

In rats with EAM, myocardial infiltration by CD45-positive cells was detectable beginning on day 14 . CD $45^{+}$cell infiltration increased prominently at day 21 before beginning a gradual decline through to day 42 (Figure 3). However, even at day 42 , increased numbers of $\mathrm{CD} 45^{+}$cells were still evident in myocardial tissues, as compared to control mice without EAM.

\section{Elevated Expression of RAGE, HMGB1, and ICAM-1 in Cardiac Tissue During Myocarditis}

Expression of RAGE, HMGB1, and ICAM-1 were all elevated in the cardiac tissue of rats with EAM. Expression of RAGE was significantly increased from day 21 onwards, but was detectable in $29 \%$ of rats beginning on day 11 (Figure 4A). Expression of HMGB1 and ICAM-1 followed a similar pattern, first becoming detectable at day 11 and then becoming sharply upregulated at day 21 (Figures 4B,C).

\section{Expression of RAGE and HMGB1 in Cardiac Myocytes and Macrophages}

Under a fluorescence microscope examination, RAGE and HMGB1 were expressed both in the cardiac myocytes and macrophages (Figure S1).

\section{Improved Cardiac Function After Blocking RAGE}

All rats with and without EAM survived through to day 42. An echocardiographic examination revealed an improved heart function on day 21 and day 42 in rats treated with sRAGE during the antigen-priming phase (Group R 0-20, Group R 0-11) when compared with untreated rats (Group C; Figure 5). However, sRAGE administration after the antigen-priming phase (Group R 12-20, Group R 21-35) failed to improve heart function relative to untreated controls at the time points examined (Figure 5).

\section{Effect of sRAGE Administration on Active Inflammation and Chronic Fibrosis}

Myocardial infiltration by CD45-positive cells on day 21 was
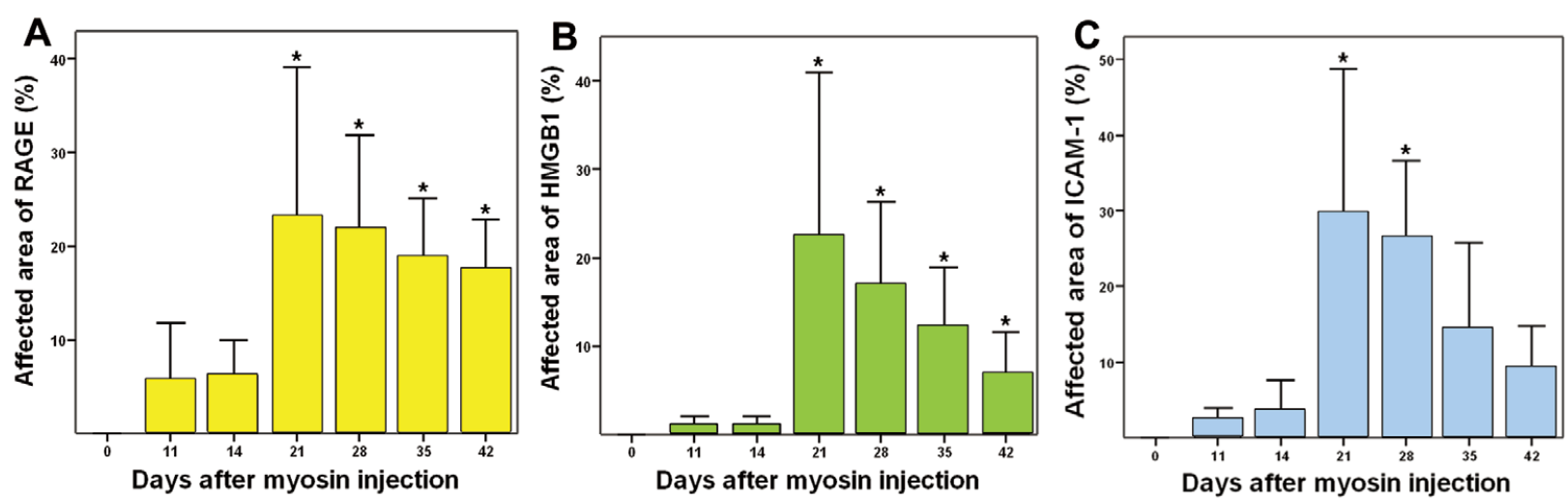

Figure 4. Expression of RAGE, HMGB1, and ICAM-1 in the heart during EAM in rats; Expression of RAGE (A), HMGB1 (B) and ICAM-1 (C) were significantly increased on day 21. ${ }^{*} \mathrm{P}<0.05$ vs day 0 . RAGE, receptor for advanced glycation end products; HMGB1, high-mobility group box 1 protein; ICAM-1, intercellular adhesion molecule 1. 

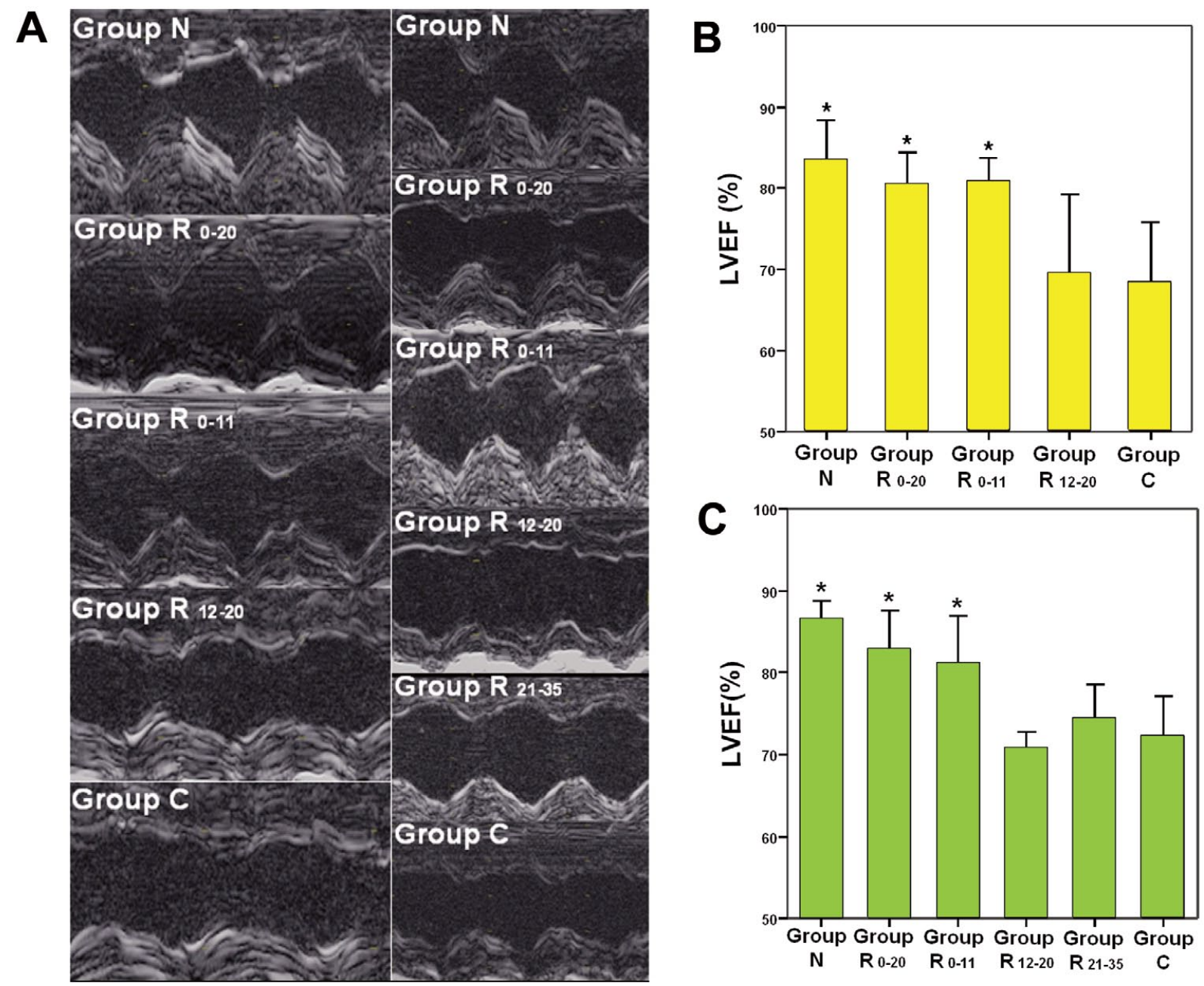

Figure 5. Heart function on day 21 and day 42 after immunization. (A) Representative M-mode echocardiogram on day 21 (Right) and day 42 (Left). Group R 0-20 (sRAGE injected from day 0 to day 20); Group R 0-11 (sRAGE injected from day 0 to day 11; Group R 12-20 (sRAGE injected from day 12 to day 20); Group R 21-35 (sRAGE injected from day 21 to day 35); Group C (untreated group); Group N (without EAM). (B) Left ventricular ejection fraction (LVEF) on day 21. (C) LVEF on day 42; rats treated during the early antigen-priming phase (Group R 0-20, Group R 0-11) presented with improved LVEF compared with untreated rats (Group C). However, sRAGE administration after the antigen-priming phase (Group R 12-20, Group R 21-35) did not improve LVEF. Data are expressed as mean+SD. *P $<0.05$ vs. Group C. sRAGE, soluble receptor for advanced glycation end products; EAM, experimental autoimmune myocarditis.

significantly attenuated in rats treated with sRAGE during the antigen-priming phase (Group R 0-20, Group R 0-11). However, in rats treated after the antigen-priming phase (Group $\mathrm{R}$ 12-20), sRAGE administration failed to attenuate the infiltration by CD45-positive cells, resulting in marked inflammation on day 21 (Figure 6). Furthermore, Masson's trichrome staining on day 42 revealed that fibrosis in Groups R 0-20 and R 0 -11 was significantly reduced compared with untreated rats (Group C). However, rats treated after the antigen-priming phase (Group R 12-20, Group R 21-35) showed a significantly larger degree of fibrosis on day 42, compared with untreated rats (Group C; Figure 6).

\section{Effect of sRAGE Administration on Myocardial T Cell Infiltration}

Myocardial infiltration by CD4- and CD8-positive cells on day 21 was significantly reduced in rats treated with sRAGE during the antigen-priming phase (Group R 0-20, Group R $0-11)$. However, in rats treated after the antigen-priming phase (Group R 12-20), sRAGE administration failed to attenuate the infiltration by CD4- and CD8-positive cells (Figure 7).

\section{Reduced Myocardial Expression of RAGE, HMGB1, and ICAM After RAGE Blocking With sRAGE}

Immunohistochemical staining of myocardium samples revealed markedly reduced expression of RAGE, HMGB1, and ICAM-1 on day 21 in those rats that received sRAGE during the early antigen-priming phase (Group R 0-20, Group R 0-11). However, there was no statistically significant difference in the expression of RAGE, HMGB1 and ICAM-1 between Group R 12-20 and Group C (Figure 8). 

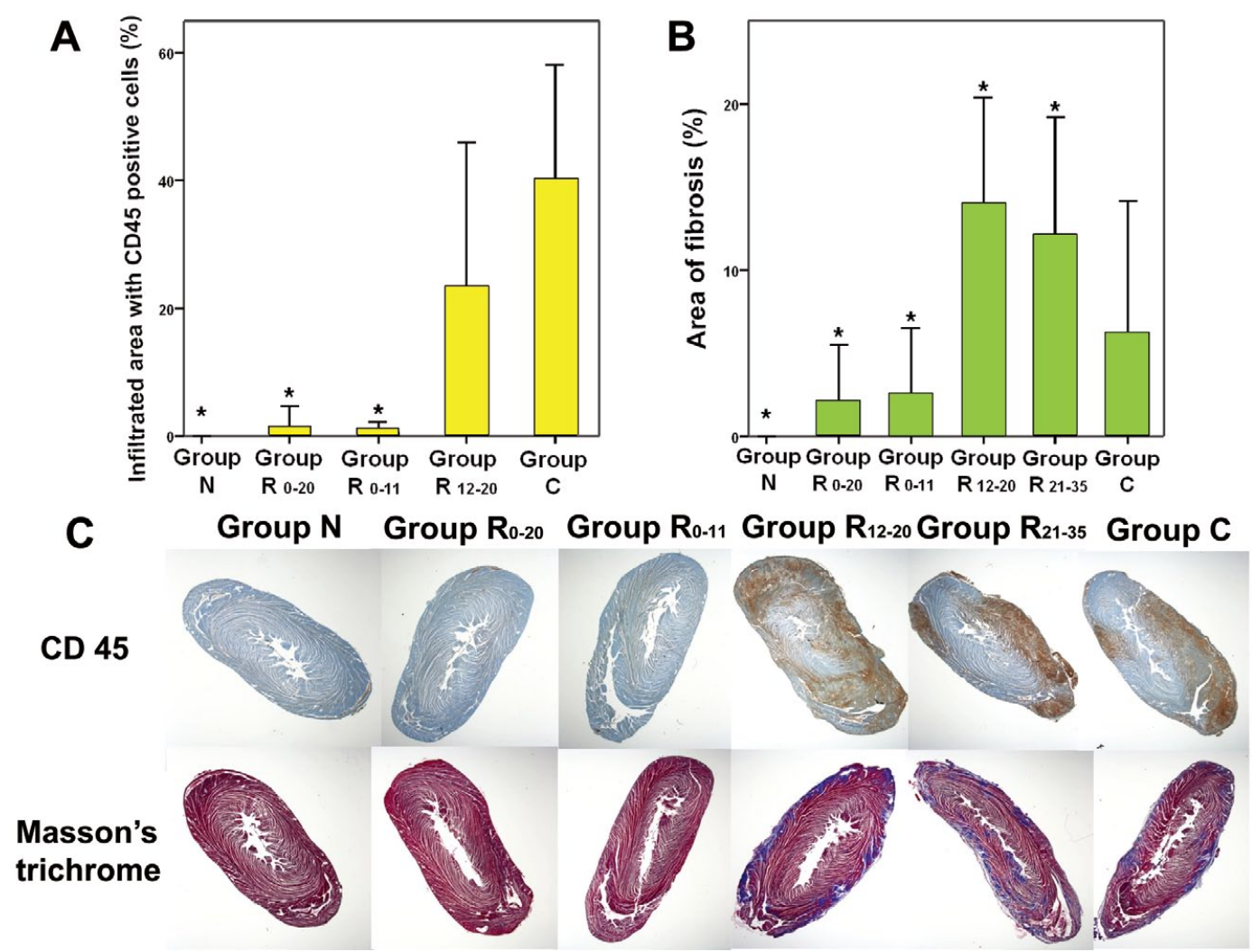

Figure 6. Acute and chronic inflammation in rats with EAM. (A) On day 21, rats injected with sRAGE during the antigen-priming phase (Group R 0-20, Group R 0-11) showed markedly attenuated CD45-positive cell infiltration, compared with untreated rats (Group C). (B) On day 42, the area of fibrosis in Groups R 0-20 and R 0-11 was significantly reduced compared with that in Group C. However, myocardial fibrosis was more prominent in Group R 12-20 and Group R 21-35 than Group C. (C) Representative images of CD45 immunohistochemical staining on day 21 and Masson's trichrome on day 42 in each group. Group R 0-20 (sRAGE injected from day 0 to day 20); Group R 0-11 (sRAGE injected from day 0 to day 11); Group R 12-20 (sRAGE injected from day 12 to day 20); Group R 21-35 (sRAGE injected from day 21 to day 35); Group C (untreated group); Group N (without EAM). Data are expressed as mean+SD. ${ }^{*} \mathrm{P}<0.05$ vs. Group C. sRAGE, soluble receptor for advanced glycation end products; EAM, experimental autoimmune myocarditis.

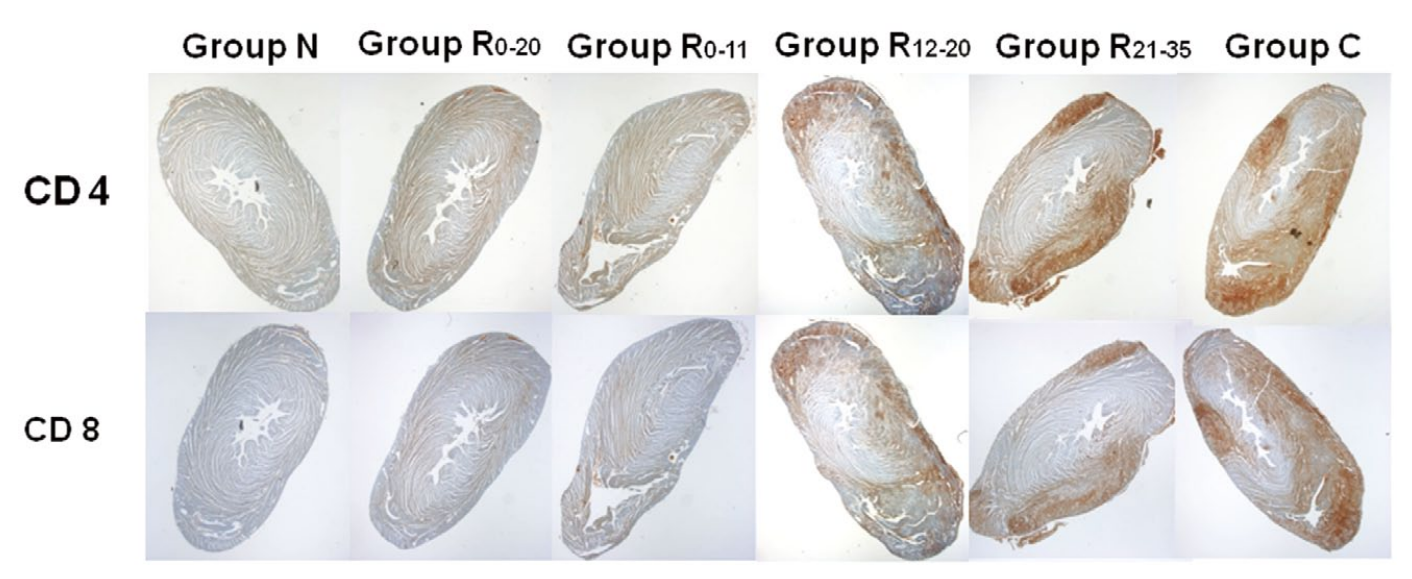

Figure 7. Representative images of CD4 and CD8 immunohistochemical staining on day 21. On day 21, rats injected with sRAGE during the antigen-priming phase (Group R 0-20, Group R 0-11) showed markedly attenuated CD4- and CD8-positive cell infiltration, compared with untreated rats (Group C). sRAGE, soluble receptor for advanced glycation end products. 

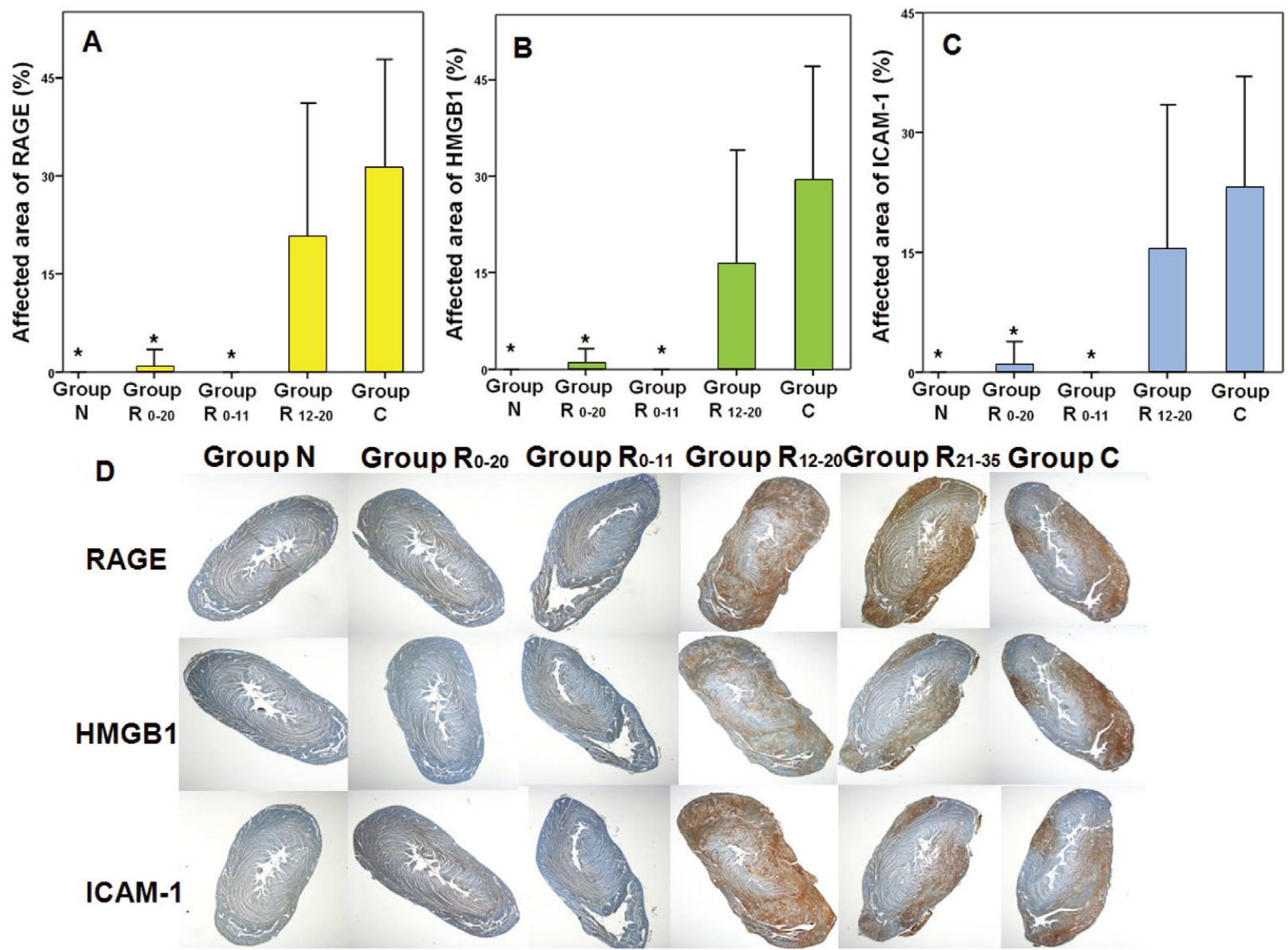

Figure 8. Expression of RAGE, HMGB1, and ICAM-1 in EAM on day 21; expression of RAGE (A), HMGB1 (B) and ICAM-1 (C) on day 21 in rats treated at the early antigen-priming phase (Group R 0-20, Group R 0-11) was significantly lower than that of untreated rats (Group C). Blocking of RAGE after the antigen-priming phase (Group R 12-20) did not significantly attenuate the expression of RAGE, HMGB1, or ICAM-1. (D) Representative images of RAGE, HMGB1, and ICAM-1 immunohistochemical staining on day 21. Group R 0-20 (sRAGE injected from day 0 to day 20); Group R 0-11 (sRAGE injected from day 0 to day 11); Group R 12-20 (sRAGE injected from day 12 to day 20); Group R 21-35 (sRAGE injected from day 21 to day 35); Group C (untreated group); Group N (without EAM). Data are expressed as mean+SD. ${ }^{*} \mathrm{P}<0.05$ vs. Group C. RAGE, receptor for advanced glycation end products; HMGB1, high-mobility group box 1 protein; ICAM-1, intercellular adhesion molecule 1; EAM, experimental autoimmune myocarditis.

\section{Discussion}

In this study, we investigated the contribution of RAGE, a member of the immunoglobulin superfamily, in the development of autoimmune myocarditis. Pathologic analysis of cardiac tissue in the rat EAM model revealed a significant increase in the expression of RAGE over controls. RAGE blockade by sRAGE during the early antigen-priming phase reduced the degree of acute and chronic inflammation and improved cardiac function. Furthermore, the early administration of sRAGE resulted in the markedly reduced expression of RAGE, HMGB1, and ICAM1 in the myocardium. However, blocking RAGE after the initial antigen-priming phase did not attenuate EAM development or reduce the expression of RAGE, HMGB1 and ICAM-1, and it failed to improve heart function.

RAGE is a multi-ligand signal transduction receptor for advanced glycation end products (AGEs), HMGB1 and S100/ calgranulin, and is an important mediator of immune respons- es. ${ }^{12,22}$ RAGE is involved in inflammation through binding ligands and subsequently activating the inflammatory cascade, as well as by promoting leucocyte recruitment as an adhesion receptor. ${ }^{22}$ Increased RAGE expression has been demonstrated in various chronic inflammatory diseases such as diabetes, atherosclerosis, rheumatoid arthritis, and inflammatory kidney disease. ${ }^{23,24}$ Although myocarditis is the most representative inflammatory disease of the heart, the role of RAGE in myocarditis has not been examined previously. This is the first reported study to demonstrate enhanced expression of RAGE in the heart during EAM and attenuation of this disease through RAGE blockade with sRAGE.

RAGE is normally expressed in lung tissue, macrophages, T cells, vascular cells, and endothelial cells. ${ }^{25}$ In this study, we found that RAGE was not significantly expressed in normal myocardium. However, in EAM, the expression of RAGE was markedly increased on day 21 during active inflammation. We also found that expression of HMGB1 was increased as well. 
This result was compatible with a recent study that demonstrated increased expression of HMGB1 in heart tissue during EAM, and an attenuation of inflammation when HMGB1 was blocked. ${ }^{26}$ HMGB1, one of the pro-inflammatory ligands for RAGE, is usually located in the nucleus, although peripheral distribution can be induced by cytokine stimulation of immune cells. ${ }^{27,28}$ The binding of HMGB1 to RAGE can influence the adhesion of inflammatory cells directly via the activation of integrins, and indirectly through the enhancement of adhesion molecule expression including ICAM-1, vascular cell adhesion molecule- 1 and tissue factors. ${ }^{29,30}$ In this study, serum levels of IL-1 $\beta$ and HMGB1 increased markedly on day 11 after cardiac myosin injection. Unlike the pro-inflammatory cytokine, IL-1 $\beta$, which remained at high levels in the serum from days 11 to 21 , the serum level of HMGB1 showed a trend toward declining between days 11 and 14, and then remained relatively constant through to day 35 (Figure 2). In contrast, the cardiac expression of HMGB1 and RAGE did not reach high levels until day 21 following auto-immunization. HMGB1 can be passively released from necrotic cells or actively secreted by inflammatory cells, such as monocytes or macrophages. ${ }^{31,32}$ The notable increase of serum HMGB1 at day 11 might be derived from secretion by inflammatory cells during the early immune process, which then induces an innate immune response via its interaction with cell surfaces receptors. Myocardial HMGB1 expression might mainly result from markedly inflamed myocardium. So, myocardial HMGB1 is likely to express late after active immune response. As HMGB1 is an important ligand for RAGE activation, it stands to reason that RAGE might be important for mediating myocarditis during early pathogenesis. This is supported by the findings presented in our current study, as we demonstrated that blocking RAGE during the early antigen-priming phase ameliorates EAM development. Furthermore, we demonstrated that blocking RAGE signaling via soluble sRAGE administration during the early antigen-priming phase led to reduced infiltration by inflammatory cells and a diminished expression of RAGE. This reduction in RAGE expression was accompanied by a reduction in the RAGE ligand, HMGB1, and the adhesion molecule, ICAM-1.

In EAM, inflammation has been demonstrated to have 3 phases: an antigen priming phase on days $0-14$, an autoimmune response phase on days 14-21, and a chronic recovery phase thereafter. ${ }^{8,20}$ During the antigen-priming phase, cardiac injury begins with the activation of the innate immune response. Next, during the autoimmune response phase, activated $\mathrm{T}$ cells are recruited to the myocardium and myocardial damage is aggravated by innate and acquired immune responses. Thereafter, the chronic recovery phase begins. To identify the kinetics of sRAGE treatment, we injected sRAGE at different times. In Group R 0-20, we injected sRAGE from day 0 to day 20 , from the antigen-priming phase to the autoimmune response phase. Rats in Group R 0-11 were injected from day 0 to day 11 , during the antigen-priming phase, while Group $\mathrm{R}$ $12-20$ was treated from day 12 to day 20 to focus on the autoimmune response phase. Treatment of Group R 21-35 occurred from day 21 to day 35 , the chronic recovery phase. On day 21, Group R 12-20 did not show any significant difference in myocardial inflammation and heart function compared with rats not treated with sRAGE. Contrary to this, Groups R 0-11 and R 0-20 presented markedly reduced myocardial inflammation. Thus, treatment with sRAGE was effective when administrated during the early antigen-priming phase.

In myocarditis, the clinical symptoms manifest after the initial phase. Therefore, therapeutic interventions need to be effective when administered after the initiating phase. Contrary to our expectations, treatment with sRAGE from day 12-20 failed to ameliorate EAM. Even though RAGE expression in the heart began in earnest after day 14, the administration of sRAGE within this phase was not able to suppress myocardial inflammation or expression of RAGE and HMGB1 on day 21. There are several possible explanations for this result. The effective dosage of sRAGE could differ according to the inflammatory phase. Alternatively, as active inflammation progresses in a vicious cycle, the amount of sRAGE required to compete for RAGE and eliminate ligands might be greater. Another possible explanation is that RAGE might be mainly involved in the early, innate immune response during myocarditis. In this study, increased HMGB1 serum levels were demonstrated at day 11 of EAM. Therefore, it is likely that the RAGE activation mediated through the HMGB1-RAGE interaction in immune cells might be important for mediating myocardial inflammation during the early phase of EAM. This might be a major reason for the lack of efficacy of sRAGE administered after day 11. Furthermore, the initial inflammation mediated by RAGE might propagate into T cell activation, and once the adaptive immune response is activated, sRAGE might not be effective at attenuating inflammation.

Contrary to our expectation, Masson's trichrome staining on day 42 revealed that rats treated after the antigen-priming phase (Group R 12-20, Group R 21-35) showed a larger degree of fibrosis on day 42, compared with untreated rats (Group C). It is not clear why myocardial fibrosis was more prominent in rats treated after the antigen-priming phase compared to the untreated rats, as the Heart function on day 42 was similar between rats treated after the antigen-priming phase and untreated rats. We do not believe it to be an effect of sRAGE blockade as rats administered with sRAGE in the antigenpriming phase did not have an increase in fibrosis.

One limitation of this study is that we could not demonstrate whether RAGE activation in the leukocytes or the cardiomyocytes was the major mechanism for the development of EAM. The co-immunostaining of RAGE/HMGB 1 with CD68 revealed that RAGE and HMBG1 were expressed both in leukocytes and cardiomyocytes. Another limitation is that we did not measure the antibody for cardiac myosin. We did not examine the effect of RAGE blocking on the production of anti-myosin antibody. Despite these limitations, we believe this study has merit in demonstrating, for the first time, the possible role of RAGE activation in the pathogenesis of acute myocarditis.

\section{Conclusions}

In conclusion, the expression of RAGE was significantly increased in the heart of EAM. The blockade of RAGE by sRAGE during the early antigen-priming phase reduced acute and chronic inflammation and significantly improved cardiac function. However, blocking RAGE after the antigen-priming phase did not ameliorate the expression of RAGE, HMGB1, or ICAM-1, and it did not reduce myocardial T-cell infiltration or improve heart function. From these results, we conclude that RAGE-mediated inflammation might be important for the initiation of myocarditis and that blockade of RAGE by sRAGE during the early phase is associated with a reduction in myocarditis and preservation of LV function.

\section{Acknowledgments}

This work was supported by a faculty research grant of Yonsei University College of Medicine for 2010 (6-2010-0169), a grant from the Korea 
Healthcare technology R \& D Project, Ministry for Health, Welfare \& Family Affairs, Republic of Korea (A085136), and the National Research Foundation of Korea (NRF) grant, which was funded by the Korean government(MEST) (No. 2010-0026367).

We thank Byung-Gon Kim and Kun-Bae Bang for their excellent technical assistance.

\section{Disclosures}

Conflict of Interest: The authors of this article reported no actual or potential conflicts of interest in relation to this study.

\section{References}

1. Cooper LT. Myocarditis. New Engl J Med 2009; 360: 1526-1538.

2. Zee Cheng CS, Tsai CC, Palmer DC, Codd JE, Pennington DG, Williams GA. High incidence of myocarditis by endomyocardial biopsy in patients with idiopathic congestive cardiomyopathy. $J \mathrm{Am}$ Coll Cardiol 1984; 3: 63-70.

3. Liu PP, Mason JW. Advances in the understanding of myocarditis. Circulation 2001; 104: 1076-1082.

4. Saji T, Matsuura H, Hasegawa K, Nishikawa T, Yamamoto E, Ohki $\mathrm{H}$, et al. Comparison of the clinical presentation, treatment, and outcome of fulminant and acute myocarditis in children. Circ J 2012; 76: $1222-1228$

5. Nakamura H, Yamamura T, Umemoto S, Fukuta S, Shioi T, Matsumori A, et al. Autoimmune response in chronic ongoing myocarditis demonstrated by heterotopic cardiac transplantation in mice. Circulation 1996; 94: $3348-3354$.

6. Rose NR, Hill SL. The pathogenesis of postinfectious myocarditis. Clin Immunol Immunopathol 1996; 80: S92 -S99.

7. Kodama M, Matsumoto Y, Fujiwara M, Masani F, Izumi T, Shibata A. A novel experimental model of giant cell myocarditis induced in rats by immunization with cardiac myosin fraction. Clin Immunol Immunopathol 1990; 57: 250-262.

8. Kodama M, Hanawa H, Saeki M, Hosono H, Inomata T, Suzuki K, et al. Rat dilated cardiomyopathy after autoimmune giant cell myocarditis. Circ Res 1994; 75: 278-284.

9. Elamm C, Fairweather D, Cooper LT. Pathogenesis and diagnosis of myocarditis. Heart 2012; 98: 835-840.

10. Schmidt AM, Vianna M, Gerlach M, Brett J, Ryan J, Kao J, et al Isolation and characterization of two binding proteins for advanced glycosylation end products from bovine lung which are present on the endothelial cell surface. J Biol Chem 1992; 267: 14987-14997.

11. Neeper M, Schmidt AM, Brett J, Yan SD, Wang F, Pan YC, et al. Cloning and expression of a cell surface receptor for advanced glycosylation end products of proteins. J Biol Chem 1992; 267: $14998-$ 15004.

12. Chavakis T, Bierhaus A, Al Fakhri N, Schneider D, Witte S, Linn T, et al. The pattern recognition receptor (RAGE) is a counterreceptor for leukocyte integrins: A novel pathway for inflammatory cell recruitment. J Exp Med 2003; 198: 1507-1515.

13. Alexiou P, Chatzopoulou M, Pegklidou K, Demopoulos VJ. RAGE: A multi-ligand receptor unveiling novel insights in health and disease. Curr Med Chem 2010; 17: 2232-2252.

14. Bierhaus A, Humpert PM, Morcos M, Wendt T, Chavakis T, Arnold $\mathrm{B}$, et al. Understanding RAGE, the receptor for advanced glycation end products. J Mol Med 2005; 83: 876-886.

15. Schmidt AM, Yan SD, Stern DM. The biology of the receptor for advanced glycation end products and its ligands. Biochim Biophys Acta 2000; 1498: $99-111$.

16. Zhang P, Cox CJ, Alvarez KM, Cunningham MW. Cutting edge: Cardiac myosin activates innate immune responses through TLRs. $J$ Immunol 2009; 183: 27-31.
17. Bucciarelli LG, Wendt T, Qu W, Lu Y, Lalla E, Rong LL, et al. RAGE blockade stabilizes established atherosclerosis in diabetic apolipoprotein E-null mice. Circulation 2002; 106: 2827-2835.

18. Taguchi A, Blood DC, del Toro G, Canet A, Lee DC, Qu W, et al. Blockade of RAGE-amphoterin signalling suppresses tumour growth and metastases. Nature 2000; 405: 354-360.

19. Yan SS, Wu Z, Zhang HP, Furtado G, Chen X, Schmidt AM, et al. Suppression of experimental autoimmune encephalomyelitis by selective blockade of encephalitogenic T-cell infiltration of the central nervous system. Nat Med 2003; 9: 287-293.

20. Okura Y, Yamamoto T, Goto S, Inomata T, Hirono S, Hanawa H, et al. Characterization of cytokine and iNOS mRNA expression in situ during the course of experimental autoimmune myocarditis in rats. $J$ Mol Cell Cardiol 1997; 29: 491-502.

21. Lang RM, Bierig M, Devereux RB, Flachskampf FA, Foster E, Pellikka PA, et al. Recommendations for chamber quantification: A report from the American Society of Echocardiography's Guidelines and Standards Committee and the Chamber Quantification Writing Group, developed in conjunction with the European Association of Echocardiography, a branch of the European Society of Cardiology. $J$ Am Soc Echocardiogr 2005; 18: 1440-1463.

22. Jandeleit-Dahm K, Watson A, Soro-Paavonen A. The AGE/RAGE axis in diabetes-accelerated atherosclerosis. Clin Exp Pharmacol Physiol 2008; 35: 329-334.

23. Bierhaus A, Stern DM, Nawroth P. RAGE in inflammation: A new therapeutic target? Curr Opin Investig Drugs 2006; 7: 985-991.

24. Park H, Baek J, Shin WS, Kim D, Jang SW, Shin DI, et al. Soluble receptor of advanced glycated endproducts is associated with plaque vulnerability in patients with acute myocardial infarction. Circ J 2011; 75: $1685-1690$.

25. Uribarri J, Cai W, Peppa M, Goodman S, Ferrucci L, Striker G, et al. Circulating glycotoxins and dietary advanced glycation endproducts: Two links to inflammatory response, oxidative stress, and aging. $J$ Gerontol A Biol Sci Med Sci 2007; 62: 427-433.

26. Su Z, Sun C, Zhou C, Liu Y, Zhu H, Sandoghchian S, et al. HMGB1 blockade attenuates experimental autoimmune myocarditis and suppresses Th17-cell expansion. Eur J Immunol 2011; 41: 3586-3595.

27. Lotze MT, Tracey KJ. High-mobility group box 1 protein (HMGB1): Nuclear weapon in the immune arsenal. Nat Rev Immunol 2005; 5: $331-342$.

28. Müller S, Ronfani L, Bianchi ME. Regulated expression and subcellular localization of HMGB1, a chromatin protein with a cytokine function. J Intern Med 2004; 255: 332-343.

29. Chavakis E, Hain A, Vinci M, Carmona G, Bianchi ME, Vajkoczy P, et al. High-mobility group box 1 activates integrin-dependent homing of endothelial progenitor cells. Circ Res 2007; 100: 204-212.

30. Orlova V, Choi EY, Xie C, Chavakis E, Bierhaus A, Ihanus E, et al. A novel pathway of HMGB1-mediated inflammatory cell recruitment that requires Mac-1-integrin. EMBO J 2007; 26: 1129-1139.

31. Scaffidi P, Misteli T, Bianchi ME. Release of chromatin protein HMGB1 by necrotic cells triggers inflammation. Nature 2002; 418: 191-195.

32. Tang D, Shi Y, Kang R, Li T, Xiao W, Wang H, et al. Hydrogen peroxide stimulates macrophages and monocytes to actively release HMGB1. J Leukoc Biol 2007; 81: 741-747.

\section{Supplementary Files}

Supplementary File 1

Figure S1. (A) Double immunofluorescence staining for RAGE and HMGB1 in the muscle and macrophages.

Please find supplementary file(s);

http://dx.doi.org/10.1253/circj.CJ-13-1235 